\title{
AN APPROACH FOR SPECIFYING THRESHOLD, REPEAT INSPECTION INTERVALS AND INSPECTION REQUIREMENTS FOR DAMAGE TOLERANT AERO-STRUCTURES
}

\author{
Ahmed Mukhtar Mohamed Ali Nour Eldin \\ PHD Student \\ Faculty of post graduates \\ Nile Valley University, Atbara, Sudan
}

\author{
M.I.Shukri \\ Professor \\ Faculty of Mechanical Engineering \\ Nile Valley University, Atbara, Sudan
}

\begin{abstract}
Damage tolerant design, aircraft inspection and maintenance are key factors for ensuring aircraft airworthiness. Damage tolerance is a property of a structure relating to its ability to sustain defects safely until repair can be achieved. Aircraft structural inspections define the size of life-limiting defects that could be present at a given time. Inspection requirements are determined by the anticipated service loads, the desired service life, and by the damage tolerance designed into the structure. This paper outlines the damage tolerance concept and how to apply this concept in design phase of the project for specifying the requirement of inspection (last phase of the DT Evaluation process), including threshold (time of the first inspection), inspection intervals (Time between 2 different inspections) and inspection type (based on clear approach for assessing the visible detectable crack length) which comply with the technical and economical requirements. The paper considers the technical concept and best practices of damage tolerance analysis and inspection program in a detailed, simplified and organized way for facilitating the process which could be considered as an approach for compliance with requirements that issued by civil regulating authorities.
\end{abstract}

Keywords - Aircraft Structure, damage tolerance, inspection program, threshold inspection, repeat interval.

\section{INTRODUCTION}

Damage tolerance technology is a discipline which when applied to aircraft structure allows safe operation of the airplane even in the event that undetected accidental, corrosive or fatigue induces damage may occur for whatever reason, within the expected operational life of the aircraft. Damage tolerance includes three major elements, residual strength of the damaged structure, crack propagation and inspection. [1] In general a damage tolerance evaluation of an aircraft structure results in an inspection program with specified inspection threshold, frequency and type. This inspection program results from an engineering evaluation considering the growth of cracks under expected in-service loading spectra starting from in-service detectable crack sizes to a crack size which would cause catastrophic failure under limit loading conditions. All conditions which effect crack growth such as the environment must be accounted for.

\section{DAMAGE TOLERANCE - AIRWORTHINESS REQUIREMENTS}

In order to develop a damage tolerant structure, certain laws and specifications must be met. All aircraft related laws and authority requirements can be found in FAR (Federal Aviation Regulations) and CS (Certifications specifications) documents; FAR for the USA, determined by the FAA (Federal aviation Administration) and CS (Certification Specifications) for Europe, regulated by EASA (European Aviation Safety Agency). For any aircraft with a maximum take-off weight (MTOW) higher than $12500 \mathrm{lbs}$, the documents needed to check are FAR-25 or CS-25. Damage Tolerance requirements appear in sections FAR-25.571 and CS-25.571, where the same goals are stated in both documents to achieve a damage tolerant design.

One of the major acceptable means for complying with damage tolerance design requirements is adequate inspection program which must show the inspection and maintenance procedures for principal structural elements that contribute significantly to the carrying of flight, ground or pressurization loads and whose integrity is essential in maintaining the overall integrity of the airplane. [2], [3], [4], [5].

\section{DEFINITION OF INSPECTION CONSIDERATIONS AND REQUIREMENTS}

The demonstrated damage tolerance of the structure must be supported by a comprehensive inspection program to ensure detection of the damage before it becomes critical. Information must be provided to allow a program to be defined for Structure Significant Item (S.S.I's) giving the required threshold and repeat inspection intervals, together with the extent of the inspection and the methods to be used. 


\section{- Threshold Inspection:}

An estimate of the fatigue strength of each critical location selected for a damage tolerance evaluation must be made. A scatter factor will be applied to this life estimate to establish the declared crack free life of the structure.

The number of flights set as the threshold interval for inspections of a particular item must be less than this declared crack free life. For areas susceptible to accidental damage the initial flaw concept will be used to determine the threshold as well as the repeat interval for inspections. [6]

\section{- Repeat Inspection Interval:}

The repeat inspection interval will be based on the crack propagation life from a defined detectable damage in the critical location of the structure to the demonstrated residual strength damage level. [6]

\section{THRESHOLD AND INSPECTION INTERVAL ASSESSMENT}

This section presents the scatter factors to be applied to fatigue and damage tolerance lives derived by calculation or obtained from a representative test (evaluation phase). These factors are used in establishing the inspection requirements for the aircraft primary structure. The factors presented are recommended factors to be used in the design phase of the project. [6]

A definition of the fatigue and crack propagation life terms used is given in figure (1) below:

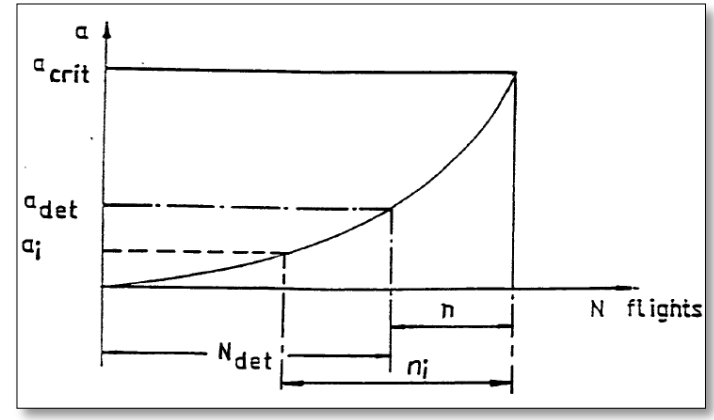

Fig (1) Definition of Fatigue crack propagation life term. [6]

\begin{tabular}{|l|l|}
\hline $\mathrm{N}_{\mathrm{C}}$ & Calculated fatigue life (flights) \\
\hline $\mathrm{N}_{\text {det }}$ & Life at detectable crack length (flights) \\
\hline $\mathrm{n}_{\mathrm{i}}$ & $\begin{array}{l}\text { Life between initial flaw and critical crack length } \\
\text { under limit loads (flights) }\end{array}$ \\
\hline $\mathrm{n}$ & $\begin{array}{l}\text { Life between detectable and critical crack length } \\
\text { under limit loads (flights) }\end{array}$ \\
\hline $\mathrm{T}_{\mathrm{DBD}}$ & Threshold inspection as defined in the DBD \\
\hline $\mathrm{I}$ & Repeat Inspection Interval (flights) \\
\hline $\mathrm{I}_{\mathrm{DBD}}$ & Repeat inspection interval as defined in the DBD \\
\hline
\end{tabular}

\begin{tabular}{|l|l|}
\hline DBD & Data Basis for Design \\
\hline
\end{tabular}

Table (1) Fatigue and crack propagation life terms. [6]

\subsection{Recommended Factors to be used in design:}

The Data Basis for Design (DBD) defines the fatigue life and inspection aims for the structure in terms of a structural endurance (design service goal) and directed inspection thresholds and repeat inspection intervals, refer to figure (2) below for getting an idea of these terms.

Entry Into Service

Fig (2) Design service goal, Inspection threshold and Inspection Interval. [3]

Table (2) below provides an example:

\begin{tabular}{|l|l|l|l|}
\hline Examples & DSG & $\begin{array}{l}\text { Inspection } \\
\text { Threshold }\end{array}$ & $\begin{array}{l}\text { Repeat } \\
\text { Inspection } \\
\text { Interval }\end{array}$ \\
\hline Global 7500 & 17000 & 8500 & 4250 \\
\hline A220 & 60000 & 30000 & 15000 \\
\hline A380 & 19000 & 7600 & 3800 \\
\hline
\end{tabular}

Table (2) Examples of DSG, inspection threshold and repeat inspection interval for transport airplanes. [3]

It is recommended that the following factors are used for establishing inspection targets (Threshold inspection and repeat Intervals). [6]:

\subsubsection{Threshold Inspection: Figure (3)}

The threshold inspection objective for directed detailed or special detailed inspection is given in the DBD. This value should not be greater than half the fatigue life design aim. [7], [8].

Threshold inspection, $\mathrm{T}$, is normally covered by $\mathrm{S}-\mathrm{N}$ calculation but it is important that consideration is given to the initial flaw concepts for regions where initial damage is likely to occur during the manufacturing process (rogue flaws)

When using S-N data for design:

Calculated fatigue life, $\mathrm{Nc} \geq 5$ Threshold inspection objective. $\mathrm{T}_{\mathrm{DBD}}$.

When using the initial flow concept it is recommended that:

I. For single load path structure, $\mathrm{n}_{\mathrm{i}} \geq 2$ Threshold inspection objective, $\mathrm{T}_{\mathrm{DBD}}$

II. For all multiple element structure, $\mathrm{n}_{\mathrm{i}} \geq 2$ threshold inspection objective, $\mathrm{T}_{\mathrm{DBD}}$ 
Where $n_{i}$ is the life between the assumed initial flaw size and the critical crack size under limit load application as shown in figure (1).

\subsubsection{Repeat Inspection Interval: Figure (4)}

The required repeat inspection objective for directed detailed or special detailed inspection is given in the data basis for design. [6]

The repeat inspection interval is normally determined by crack propagation and residual strength analysis, although consideration may be given to probabilistic analysis.

For design the crack propagation life (n) repeat inspection interval, $\mathrm{I}_{\mathrm{DBD}}$

I. For single load path structure $n \geq 3$ Repeat Inspection Interval, $\mathrm{I}_{\mathrm{DBD}}$

II. For multi-element structure, where inspectable crack length is less than one load path failure $n \geq 2$, I

III. For multi-element structure, where inspectable crack length is not less than one load path failure. $\mathrm{n} \geq 3 \mathrm{I}_{\mathrm{DBD}}$

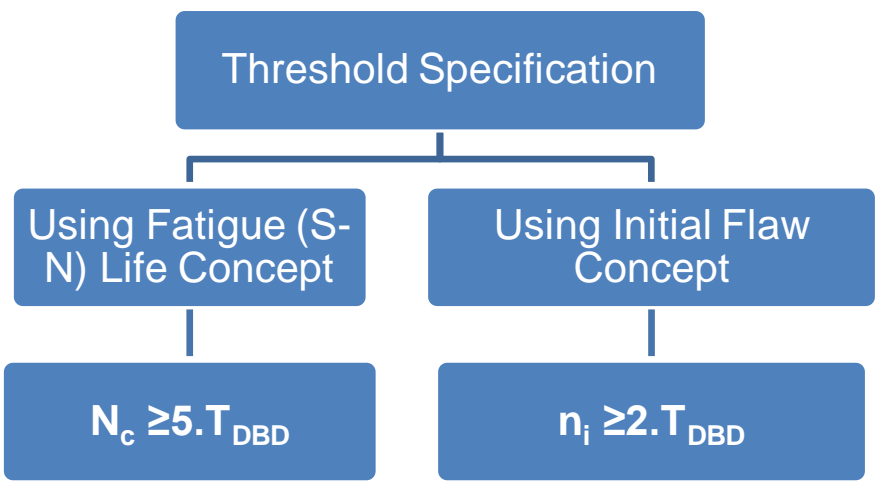

Fig (3) Scatter Factors to be applied to threshold Specifications for Design. [6]

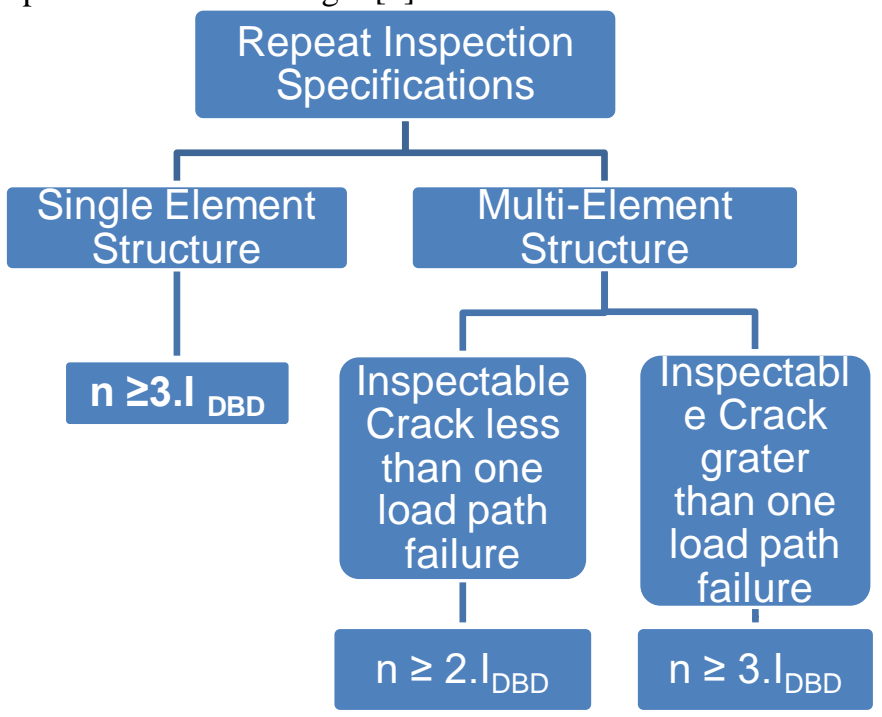

Fig (4) Scatter Factors to be applied to repeat Inspection specifications for Design. [6]

\section{SPECIFYING TYPE OF INSPECTION}

Two inspection programs are used for the surveillance of the structure:

a) The Zonal inspection program for general surveillance.

b) The directed Inspection Program.

The following term is essential in maintenance program; accordingly clear definition is stated in next paragraphs.

- Inspection: An examination of an item against a specific standard.

- Inspection- Detailed:

An intensive visual examination of a specified detail, assembly or installation. It searches for evidence of irregularity using adequate lighting and where necessary, inspection aids such as mirrors, hand lens, etc. Surface cleaning and elaborate access procedures may be required. [9], [10]

\section{- Inspection - General Visual:}

A visual examination that will detect obvious unsatisfactory conditions/discrepancies. This type of inspection may require removal of fillets, fairings, access panels/doors, etc. work stands, ladders. Etc. may be required to gain proximity. [9], [10]

\section{- Inspection - Special Detailed:}

An intensive examination of a specific location similar to the detailed inspection except for the following differences. The examination requires some special technical technique such as non-destructive test techniques, dye penetrant, high powered magnification, etc... and may require disassembly procedures.

\section{- The Zonal Inspection Program:}

The zonal inspection program is composed of general visual inspection tasks for each zone of the aircraft (surveillance of systems, power plant and structure for general conditions)

The inspections are specified by quoting the relevant zone number, zone description, access and inspection interval required. [9], [10]

The inspection tasks do not provide a description of all items to be inspected within each zone because the inspector is supposed to have an adequate level of knowledge of the aircraft design.

\section{- The Directed Inspection Program:}

In case that the zonal program is not adequate to ensure the safety of the aircraft, a specific and precise structure 


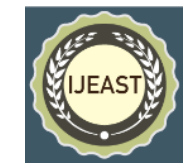

maintenance program is defined using the "Directed Inspection Program”. [9], [10]

Two inspection levels are used:

- The detailed inspection corresponding to a detailed visual inspection as defined in the definitions.

- The special detailed inspection, using special techniques where necessary (e.g NDT)

The units to define the inspection interval are:

a) Flights (for fatigue surveillance)

b) Calendar time or flight hours (for environmental surveillance)

c) Flights or calendar time (for accidental damage surveillance, dependent on the consequences to be considered.

Inspection threshold and repeat intervals for directed inspections: (Example A-320)

Fatigue damage surveillance

- Threshold: $20000 \mathrm{~F}$

- Repeat interval: $7500 \mathrm{~F}$ and $15000 \mathrm{~F}$

Environmental damage surveillance:

- 8 Years

- 4 years and 8 years.

\section{SELECTION LEVEL OF INSPECTION}

The objective is to select the lowest level of inspection, which is practicable, economical and effective from the following:
a. General visual inspection
b. Detailed inspection
c. Detailed special inspection.

Refer to figure (5) which describes the process of selecting the level of inspection, the process starts with preparation phase and evaluation phase of damage tolerance evaluation and then transfer to selecting the level of inspection, if the visual inspection is possible it will be efficient and effective from economical point of view to go forward to select the lowest level in visual inspection itself, based on specific methods which will be described later, detectable crack size of this level is determined to be as an input for specifying the threshold and repeat interval, then decision should be taken to adopt this level of inspection or to go to the higher level based on applicability and effectiveness of calculated intervals and threshold. In case the visual inspection is not possible or applicable, NDT methods should be considered as a main choice. Same process for calculating minimum detectable crack size for selected NDT method should be followed to check the applicability, efficiency and effectiveness of the threshold and repeat intervals. [11]

\subsection{Assessment of Detectable Crack length by visual Inspection:}

For determining the detectable crack length in case of visual inspection, the following method is applied. In this method the following parameters are considered:

- Level of inspection

- Access to view

- Size of item

- Lighting condition

- Surface conditions

- Material thickness and edge effect

- Hidden crack length.

The flow diagram shown in figure (6) shows the method used to determine the detectable crack length. [6]

\subsection{Selection of ratings for each parameter}

\section{a. Viewing Rating:}

This is governed by how close the eye is to the item being inspected, the distance being graded as follows, (table 3) below.

When general visual inspection is considered then the viewing rating should be selected according to the zonal inspection condition.

When detailed inspection is considered a viewing rating of 3 must be selected.

\begin{tabular}{|l|l|}
\hline 0 & $\begin{array}{l}\text { No access - Hidden item or distance greater } \\
\text { than } 3 \text { meters }\end{array}$ \\
\hline 1 & $\begin{array}{l}\text { "Poor" when the distance is 1.5 meter to 3 } \\
\text { meters. }\end{array}$ \\
\hline 2 & $\begin{array}{l}\text { "Moderate" when the distance is 0.5 meter to } \\
1.5 \text { meter }\end{array}$ \\
\hline 3 & $\begin{array}{l}\text { "Good" when un-restricted, as close as } \\
\text { needed }\end{array}$ \\
\hline
\end{tabular}

Table (3) Viewing Rating. [6]

\section{b. Congestion rating:}

Congestion is divided into three conditions depending on the number of components in the area to be inspected and the complexity of the equipment. They are to be judged as follows:

\begin{tabular}{|l|l|}
\hline 1 & Congested \\
\hline 2 & Moderate congestion \\
\hline 3 & Clear area \\
\hline
\end{tabular}

Table (4) Congestion Rating. [6] 
Whatever inspection level is considered, general visual or detailed, congestion rating should be selected in accordance with the SSI (Significant Structural Item) area.

\section{c. Size Rating:}

The size of the item or area to be inspected is to be assessed as follows:

Size of the zone:

- Large area: such as complete skin in the case of the fuselage or wing.

- Medium area: for approximately a square meter or less.

Size of SSI:

- "Large fitting" such as frames, spar, etc.

- "Medium size fittings" to include portions of frames, spars, ribs and intercostals or stringers, etc.

- "Small fittings" items of not more than approximately 10 centimeters square.

The size rating is obtained from the following table:

\begin{tabular}{|l|l|}
\hline Rating & Item/ Area Size \\
\hline 1 & Large area \\
\hline 2 & Medium area/large fittings \\
\hline 3 & Medium size fittings \\
\hline 4 & Small area \\
\hline
\end{tabular}

Table (5) Size Rating. [6]

General visual inspection requires an assessment based upon the size of the zone as described in the zonal program, whereas for a detailed visual inspection, the real size of the concerned SSI has to be taken for assessment.

\section{d. Lighting rating:}

Lighting conditions and quality will vary from bright sunlight to the use of a torch and mirror. The three ratings considered are to be associated with the type of inspection involved.

\begin{tabular}{|l|l|}
\hline 1 & $\begin{array}{l}\text { Exterior of aircraft shadow e.g. landing gear bay } \\
\text { without directed light }\end{array}$ \\
\hline 2 & $\begin{array}{l}\text { Exterior aircraft in full daylight, inside aircraft } \\
\text { with artificial light }\end{array}$ \\
\hline 3 & Concentrated lighting as required. \\
\hline
\end{tabular}

Table (6) Lighting Rating. [6]

Rating 1 and 2 should be used for general visual inspection (normal cabin light can be used as necessary)

Rating 3 is to be used for detailed visual inspection.

\section{e. Surface Rating:}

Surface conditions will vary according to the nature of the paint, presence of sealant and cleanliness.

Two ratings are considered as follows:

\begin{tabular}{|l|l|}
\hline 1 & $\begin{array}{l}\text { Areas or items liable to be covered in sealant } \\
\text { or subjected to excessive grease, oil or dirt } \\
\text { contamination }\end{array}$ \\
\hline 2 & Clean areas \\
\hline
\end{tabular}

Table (7) Surface Rating. [6]

Detailed inspections to be rating as 2 , due to preparation of surface before inspection.

The basic detectable crack length, $\mathrm{L}_{\mathrm{BAS}}$, can now be obtained from the chart on figure (7) by inserting the feasibility rating number and reading off the basic visible detectable length.

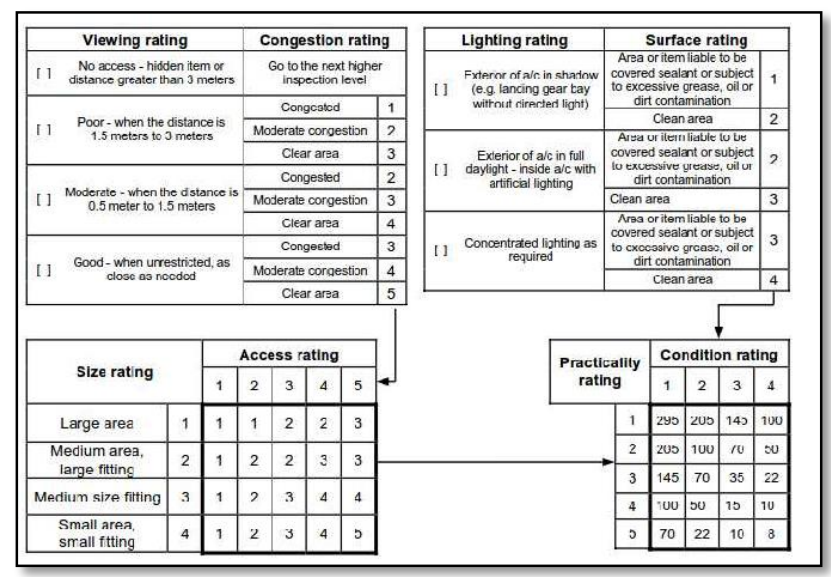

Fig (7) Chart for determining Basic detectable crack length. [3]

\subsection{Effective Detectable Crack length:}

Two effects should be considered and applied for basic detectable crack length to get the effective detectable crack length refer to figure (6), the effects are:

- Material gauge effect

- Edge effect

\subsubsection{Visible Detectable Crack length: $\left(\mathrm{L}_{\mathrm{vis}}\right)$}

The basic detectable crack length, $\mathrm{L}_{\mathrm{BAS}}$, is obtained from the chart on figure (7) by inserting the feasibility rating number and reading off the basic visible detectable length.

\section{a. adjustment for material gauge effect:}

The thicker material of an item, the tighter a crack is held together and, therefore the more difficult is to detect. To cater for this the basic detectable crack length is adjusted as shown in table below: 
International Journal of Engineering Applied Sciences and Technology, 2020

Vol. 5, Issue 3, ISSN No. 2455-2143, Pages 107-115

Published Online July 2020 in IJEAST (http://www.ijeast.com)

\begin{tabular}{|l|l|}
\hline $\begin{array}{l}\text { For material thickness less } \\
\text { than } 5 \mathrm{~mm}\end{array}$ & multiply X 1 \\
\hline $\begin{array}{l}\text { For material thickness n } 5 \\
\text { mm to } 10 \mathrm{~mm}\end{array}$ & multiply X 1.25 \\
\hline $\begin{array}{l}\text { For material thickness } \\
\text { greater than } 10 \mathrm{~mm}\end{array}$ & multiply X 1.5 \\
\hline
\end{tabular}

Table (8) Material Gauge Effect Adjustment. [6]

\section{b. adjustment for edge effect:}

For cracks which originate from or terminate at the edge of a member, the detectable length is to be multiplied by 0.5 . For non edge cracks multiply by 1 .

The visible detectable crack length is defined as follow:

$$
L_{V I S}=L_{B A S} \times(\text { gauge Factor }) \times(\text { edge Factor })
$$

Where: $\mathrm{L}_{\mathrm{BAS}}$ is basic detctable crack length

\subsubsection{Effective Detectable Crack Length: $\left(\mathrm{L}_{\mathrm{DET}}\right)$}

The visible detectable crack length after adjustment is determined for visual inspection only and does not take account of any hidden length of cracks.

When determining the propagation time from detectable length to critical length this hidden portion of the crack is to be considered. The effective detectable crack length is defines as follows:

$$
L_{D E T}=L_{V I S}+L_{H}
$$

$$
\begin{aligned}
& \text { Where: } \\
& \boldsymbol{L}_{\boldsymbol{D E T} T} \text { is effective detectable crack length } \\
& \boldsymbol{L}_{V I S} \text { is visible detectable crack length } \\
& \boldsymbol{L}_{\boldsymbol{H}} \text { is hidden crack length }
\end{aligned}
$$

\subsubsection{Hidden Crack Length: $\left(\mathrm{L}_{\mathrm{H}}\right)$}

The determination of the hidden crack length is dependent on many factors including: design, local loading and material selection. Thus, its determination can only be accurately performed by the stress office of the manufacturer. Figure (8) below shows a part of the cracked element can be hidden by fasteners, rivets, supports, brackets...etc.

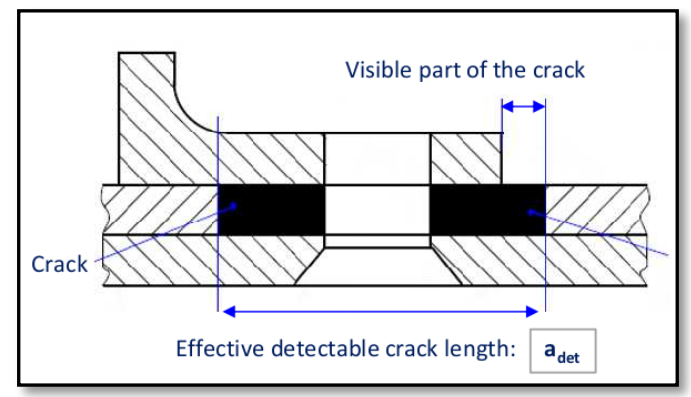

Fig (8) Hidden crack length. [3]

\subsection{Assessment of Detectable Crack length for Special Detailed Inspection (NDT):}

With reference to figure (5), when detailed visual inspection is not appropriate, the use of NDT methods has to be considered to determine if the detectability can be improved sufficiently to achieve practical inspection intervals.

In this case, considering the crack propagation curve, it is necessary to determine the crack length which should be detected to obtain the appropriate inspection interval. [12], [13].

The most appropriate NDT method has to be selected considering the following:

- The desired detectable crack length.

- The geometry and materials of the SSI

- The probable damage locations.

Once a NDT method has been selected, its qualification will have to be done to confirm the effectiveness of the selected task. Figure (9) below depicts probability of detection for non destructive test methods that used in inspection. [12], [13]

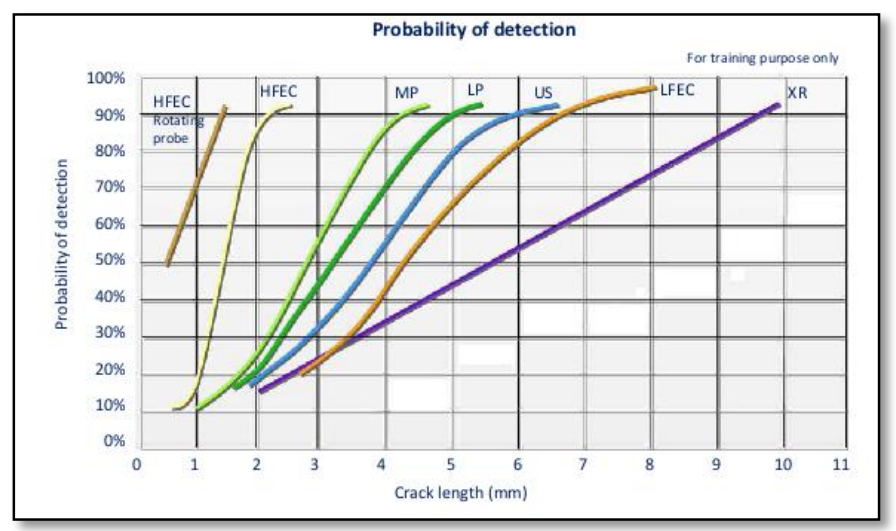

Fig (9) NDT Methods, Probability of Detection. [3]

\section{CONCLUSION}

Through the paper, detailed approach for specifying threshold, inspection interval was introduced with highlighting the best practices that followed by aircraft manufacturers for issuing structural maintenance program. As one of the main objectives of damage tolerance design, inspection program should be clearly defined based on technical analysis, test results and experience that gathered through different projects. Feedback from operators of the product could be great input for justifying or tuning of inspection program. [16]. this paper could be a reference for students, engineers, design organizations and maintenance organization for understanding the approach of specifying the inspection program for the aircraft. 


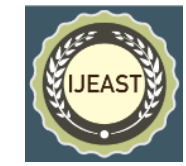

\section{Damage Tolerant SSI}

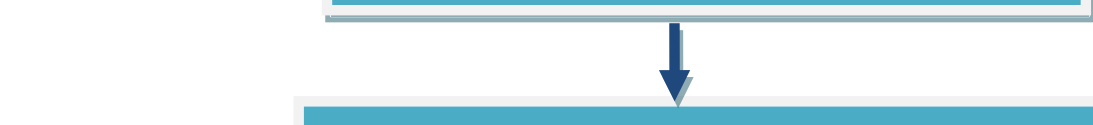

Fatigue Life Crack Propagation Characteristics

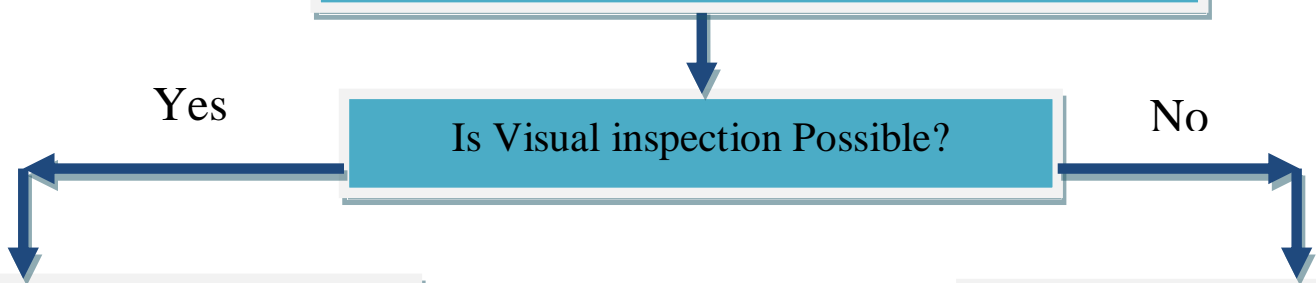

Select Lower Inspection Level

Determine Detectable Crack length

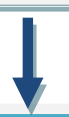

Determine:

- Threshold

- Repeat Inspection Interval
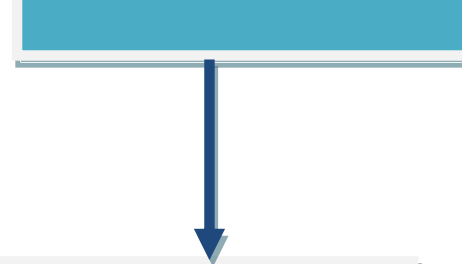

Is this interval practicable and effective?

Yes

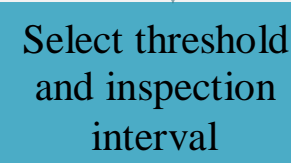

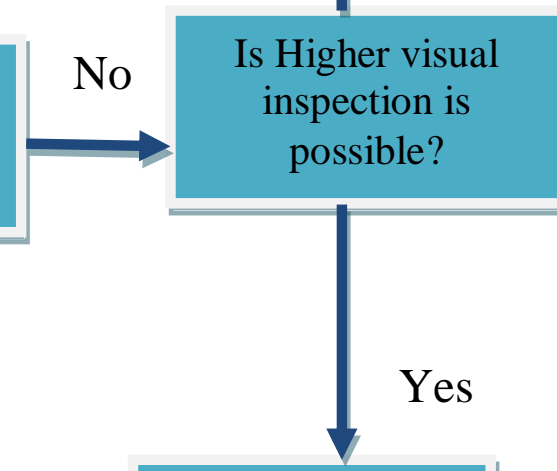

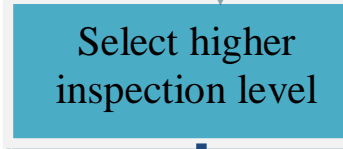

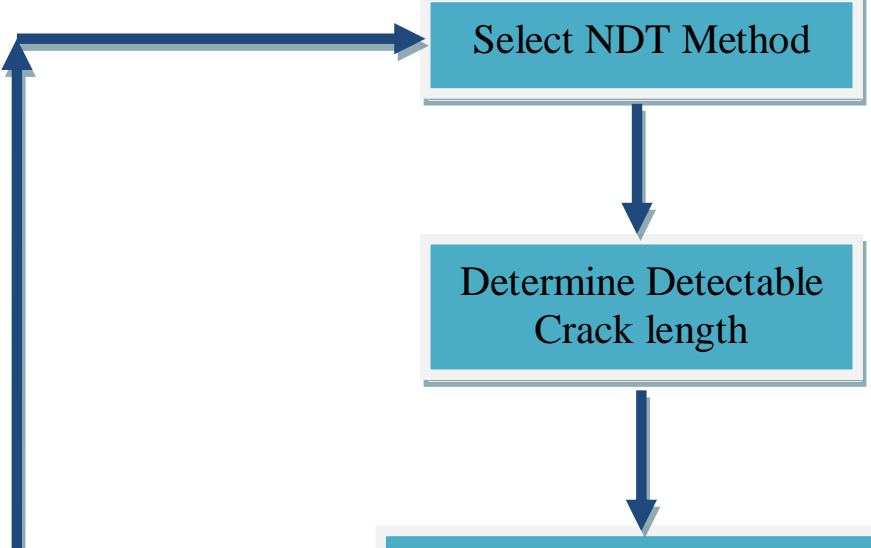

Determine:

- Threshold

- Repeat Inspection Interval

No

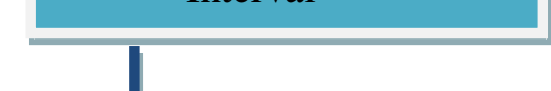

Yes

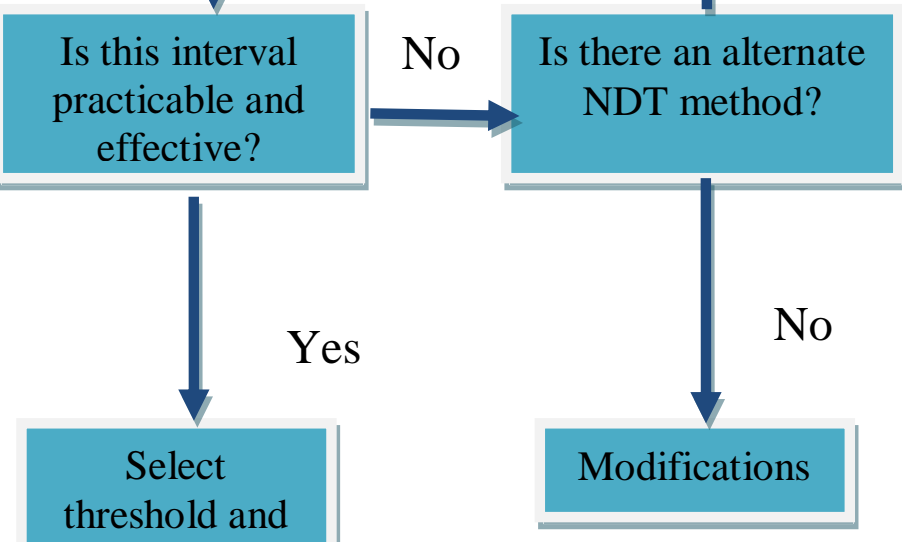

Fig (5) Selection the level of Inspection. [6] 
International Journal of Engineering Applied Sciences and Technology, 2020 Vol. 5, Issue 3, ISSN No. 2455-2143, Pages 107-115

Published Online July 2020 in IJEAST (http://www.ijeast.com)

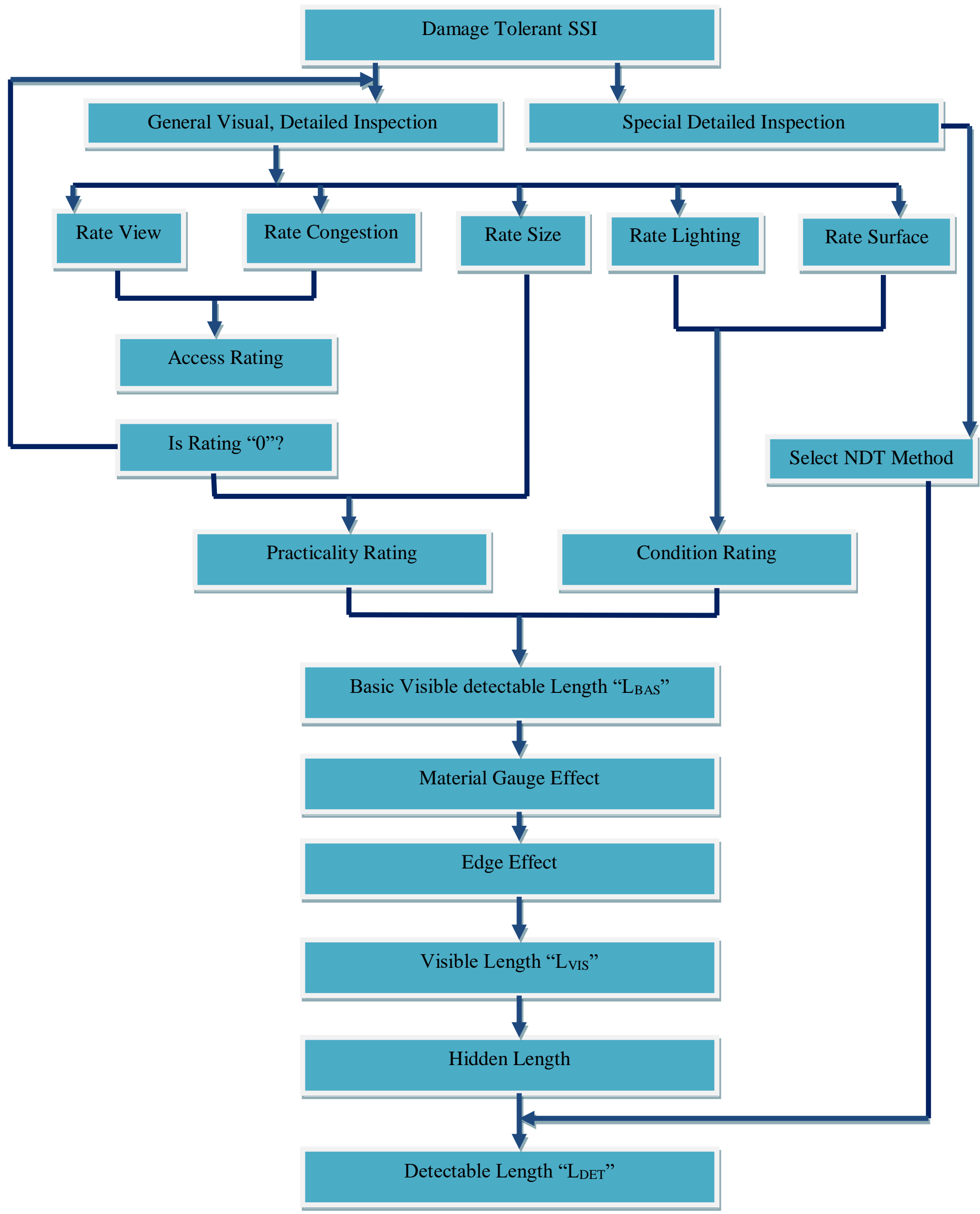

Fig (6) Assessment of Detectable Crack Length - Metallic Structure. [6] 


\section{REFERENCES}

1. T. Swift. (2000). Damage Tolerance Capability, Federal Aviation Administration, Long Beach, CS, USA (pp. 5-11)

2. NIU, M. C.-Y. (1997). Airframe Stress Analysis and Sizing. [S.1.]: Hong Kong Conmilit Press, (p. 660-668)

3. Didier Duprat. (2019) Practical Fatigue and Damage Tolerance for Aircraft. First edition. (pp.87-103, 231-258)

4. Certification Specifications for Large Aeroplanes CS-25, Amendment 3, 19 September 2007, Annex to ED Decision 2007/010/R, paragraph 25.571, Damage Tolerance and Fatigue Evaluation of Structure. European aviation Safety Agency. (pp. 1-C-22, 1-C-23, 1-C-24).

5. FAA Advisory Circular (AC) 25.571-1B. Damage Tolerance and Fatigue Evaluation of Structures, 18. Feb. 1997. (pp. 4-13)

6. Airbus Fatigue and Damage Tolerance Guidelines. (pp. B2-B18, DII4-DII9, F2-F18, GI1-GVI5)

7. T. Swift. (2000) . Damage Tolerance Technology - stress analysis oriented fracture mechanics, Hamburg, Germany. (pp. 10.3-10.15)

8. Brussat, T.R., Kathiresan, K. \& Rudd, J.L. (1986). Damage Tolerance assessment of Aircraft Lugs, Engineering Fracture Mechanics, Vol. 23, No. 6, pp. 1067-1084. (pp.11-17)

9. USAF damage tolerance design guidelines as specified in the Joint Service Specification Guide (JSSG-2006). (pp.419)

10. T. Swift. (1981) Application of Damage Tolerance Technology to Type Certification, SAE Technical Paper Series 811062. (pp. 2-5)

11. T. Swift. (2003). Fail Safe design requirements and Features, Regulatory requirements, AIAA Series 2783. (pp. 1-5)

12. Advisory Circular No. 43-204, Visual Inspection for Aircraft, Federal Aviation Administration. (pp. 43-52)

13. DOT/FAA/AR-96/65, Visual Inspection Research Project Report on Benchmark Inspection, federal Aviation Administration, September 1996. (pp. 26-60)

14. A. Arrieta and A. Striz. (2000). Optimal Design of Aircraft Structures with Damage Tolerance Requirements, AIAA 2000-4925.

15. F. C. Campbell, Inspection of Metals, Understanding the Basics, Chapter 1, Inspection Methods, Overview and Comparison. (pp. 1-20)

16. Padmaja Reddy K, Bhargav Yandra, S S Subramanya Sastry, Fatigue and damage Tolerance Analysis of Aircraft Structural Components, CYIENT, www.cyient.com. (pp.115) 